\title{
Control Architecture for Increased Performance in Pointing Lasers
}

\author{
Dr. Salvatore Cusumano Dr. Lawrence Robertson, and Lt. Jason Tellez \\ Air Force Research Lab \\ Directed Energy Directorate, ABL Technologies (AFRL/DEBA) \\ 3550 Aberdeen Ave., SE \\ Kirtland AFB, NM 87117 \\ cusumaos@plk.af.mil, robertsl@plk.af.mil, tellezj@plk.af.mil \\ (505) 846-0463, (505) 846-7687, (505) 846-4105 \\ Dr. Charly Tipton, David Jordan, Keith Ratte \\ Dynacs Engineering \\ 201 Eubank NE \\ Albuquerque, New Mexico 87123 \\ tiptonc@plk.af.mil, jordand@plk.af.mil
}

(505) 846-6355

\begin{abstract}
An overview of a control's architecture for pointing a laser in a track loop that performs with low frequency, type II, error rejection without the use of two free integrators in the forward path is presented. The method uses a stabilization loop on the outgoing laser to follow the residual error of the track loop. The cost of this loop is the addition of a position sensor and correction mirror. These components are normally used in high-performance pointing systems, thus the cost is only the delta increase in requirements from a conventional loop to that proposed. Additional effort is needed to calibrate the track sensor and stabilization sensor such that errors can be cross referenced. This capability allows for the additional error rejection between the two loops. The ability to accurately calibrate these detectors limits the performance of the total loop interaction. Initial simulation results show excellent type II error rejection without the overshoot typical in classical type II error rejection loops.
\end{abstract}

\section{TABLE OF CONTENTS}
1. INTRODUCTION
2. CONCEPT AND THEORY
3. SIMULATION
4. REPRESENTATIVE REQUIREMENTS
5. PROPOSED EXPERIMENTS
6. SUMMARY

\section{INTRODUCTION}

In laser pointing applications, alignment of the outgoing beam, hereto referred to as the pointer, with the tracking system line-of-sight (LOS) is a difficult problem and always requires ingenuity to achieve reliable performance. Optics not common to both the pointer and the target tracking system can be subjected to different vibration, acoustic and wavefront environments which in turn create a pointing error between the track LOS and the pointer LOS.

Traditionally, optical designers have attempted to minimize the non-common paths of the tracker and the pointing laser. This design approach, however, has only been satisfactory in achieving errors on the order of a few microradians [1]. A development by Lockheed Martin Missiles and Space Corporation significantly improves the standard optical architecture for pointer insertion and potentially provides submicroradian performance for dynamic rejection of disturbances. The approach reduces non-common path dynamic disturbances and also provides the additional benefit of a type-two error rejection transfer function on disturbances that enter the track loop.

This concept uses target track errors and laser pointer errors from cross calibrated sensors. The additional complexity includes the precision calibration required to align these sensors and the increased bandwidth for the pointer stabilization loop. Simulation results are encouraging and show a type II error rejection for the outgoing pointer without the commonly expected overshoot due to phase loss for these types of loops. The Common Path Common Mode (CPCM) control architecture, invented at Lockheed Martin Missiles and Space [2], was originally used to explain performance with reference to operational amplifier architectures which rejected the common mode disturbances on the positive and negative inputs. 


\section{CONCEPT AND THEORY}

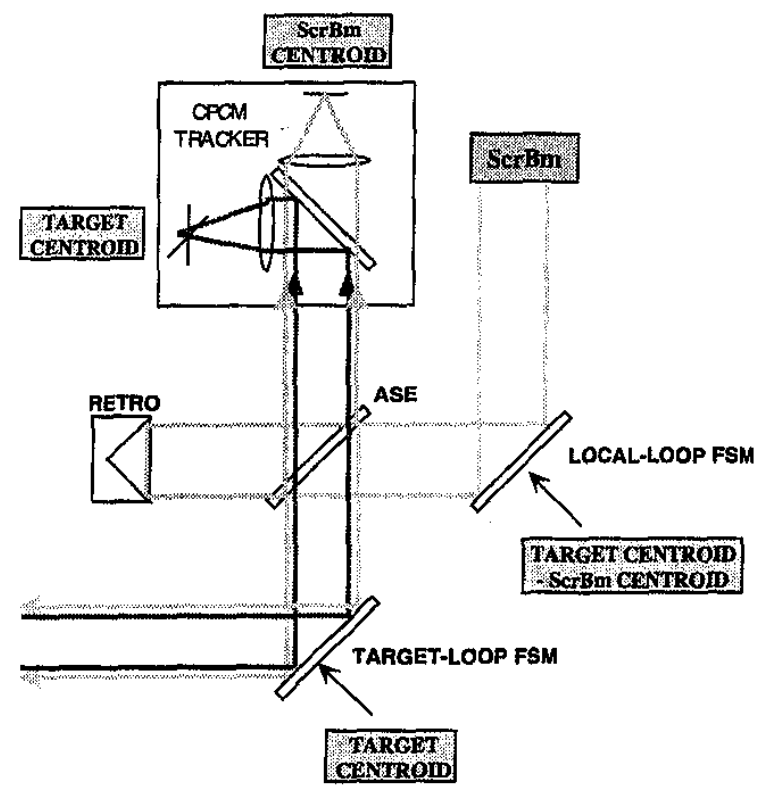

Figure 1. CPCM Concept

The basic concept of CPCM is shown in Figure 1. The target tilt is estimated from the track camera's measurement of the target centroid. The Target-Loop Fast Steering Mirror (FSM) corrects the LOS disturbances with tilt errors sensed by the tracker. The pointer/Scoring Beam (ScrBm) is inserted into the optical system with the use of the Aperture Sharing Element (ASE).

The path from the ASE to the target (considered 'downstream' of the ASE) is common to the tracker LOS and the ScrBm LOS. Any disturbances sensed along this path would be corrected up to the performance of the target tracker. This is the fundamental operation of a conventional pointer-tracker. However, an assumption is made that once the $\mathrm{ScrBm}$ /pointer is aligned to the tracker LOS, no further $\mathrm{ScrmBm}$ correction relative to the target loop is necessary. For a type 1 track loop, the rejection of disturbances entering down stream of the ASE would show a characteristic $20 \mathrm{db}$ roll off. Since this setup would not reject disturbances that affect only the ScrBm, some conventional pointer-trackers include a local stabilization loop on the $\mathrm{ScrBm}$ /pointer laser, which uses information from only the pointer camera. This loop rejects disturbances to the pointer LOS that occurred 'upstream' of the ASE, i.e., between the ASE and the pointer's laser source. This loop corrects for thermal drift of the laser source or seismic disturbances that usually are present. The local loop improves pointing since microradian disturbances are present in most applications of pointer-trackers. However, the local loop only helps with pointer stability and in no way changes the error rejection for the critical LOS path to the target.

Figure 2 show how CPCM works. The two loops are connected so that the pointer is now referenced to the tracker. The local loop for the pointer then moves directly to the proper aimpoint on the target. CPCM can be implemented in any system in which an outgoing beam must be aligned with target optical paths.

CPCM implements the track loop and local loop in the conventional manner but then adds an additional correction path in the loop architecture. This is best seen with reference to Figure 3. The block diagram depicts the essential elements of a pointer-tracker problem assuming that most of the dynamics are in $\mathrm{Gt}$ and $\mathrm{Gp}$. For the conventional architecture Switch $A$ is open. The error rejection for target dynamics $\left(\mathrm{U}_{\mathrm{d}}\right)$ to pointer jitter on the target $\left(\mathrm{Y}_{\mathrm{t}}\right)$ is simply (using control block diagram logic),

$$
\frac{Y_{\mathrm{t}}}{U_{d}}=\frac{1}{(1+G)}
$$

In deriving (1) the tracker and local loop sensors were assumed to have dynamics well above the closed-loop bandwidth. This is an excellent assumption for most pointer-tracker problems. If the tracker error is now used to drive the local stabilization loop for the pointer, (2) is derived for the same conditions assumed to calculate (1).

$$
\frac{Y_{t}}{U_{d}}=\frac{1}{(1+G)^{2}}
$$

In deriving (2) the assumption that the tracker and local loop had the same bandwidths was made, i.e., $G=G_{t}=G_{p}$ which is not uncommon. The performance improvement is now readily apparent

CPCM does, however, require additional cost and effort to implement. First, CPCM adds more complexity to the overall control system. Instead of having a single FSM to point the beam, CPCM requires two FSMs - one for the target LOS another for the pointer stabilization loop. The sensors for these loops must also be cross calibrated so that tilts measured in each can be combined for the delta correction into the pointer stabilization loop. This adds to software development, integration and test. (We implicitly assume that the loop is implemented with digital controllers. This need not be the case.) Software complexity should never be underestimated and is the more than likely the primary additional cost to the system. The inclusion of the additional hardware for the stabilization loop is not insignificant, but is less than the cost of the software. Second, CPCM requires precision optical elements for the 
pointer sensor. The additional hardware costs for CPCM are estimated as modest over standard pointer stabilization loops since all of the essential optical elements are already in place. However, the additional complexity and time to test and integrate CPCM should be well examined before it is used.

\section{SIMULATION}

CPCM was simulated to verify performance qualities. Figure 3 shows the CPCM simulation block diagram. An open

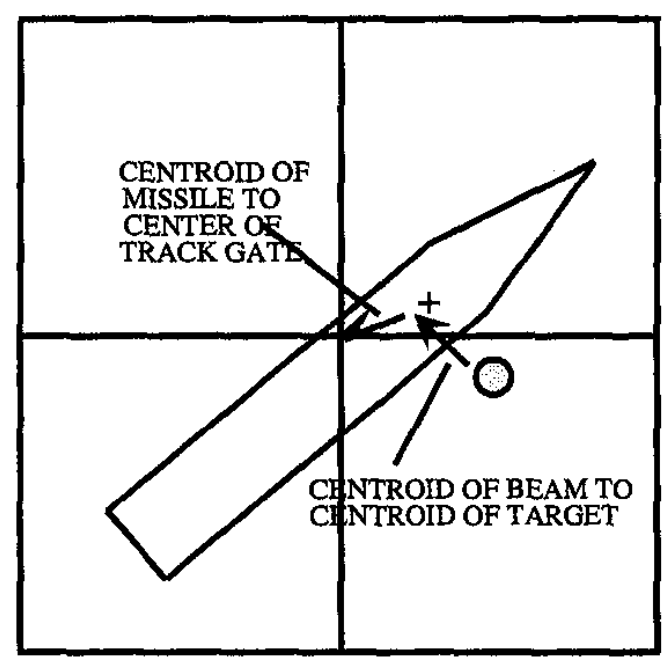

loop tilt error was created using known Power Spectral Density (PSD) atmospheric spectra converted to a time series and than added to the track camera Noise Equivalent Angle (NEA). A camera latency was then added to the time series data and multiplied by both the closed loop bandwidth of a track mirror and the $\mathrm{ScrBm} /$ pointer steering mirror closed loop response. The output of the beam steering mirror is subtracted by the output of the target track tilt error to simulate the CPCM effect of the outgoing beam. The jitter at the far field is found by adding the atmospheric tilt effects back into the outgoing ScrBm.

Figures $4 \mathrm{a}$ and $4 \mathrm{~b}$ show the closed loop error rejection with and without, respectively, the track error subtracted in the

\section{CPCM TRACKING}

- Target centroid is conventional

- Beam centroid referenced to target centroid is unconventional

Figure 2. CPCM Tracking

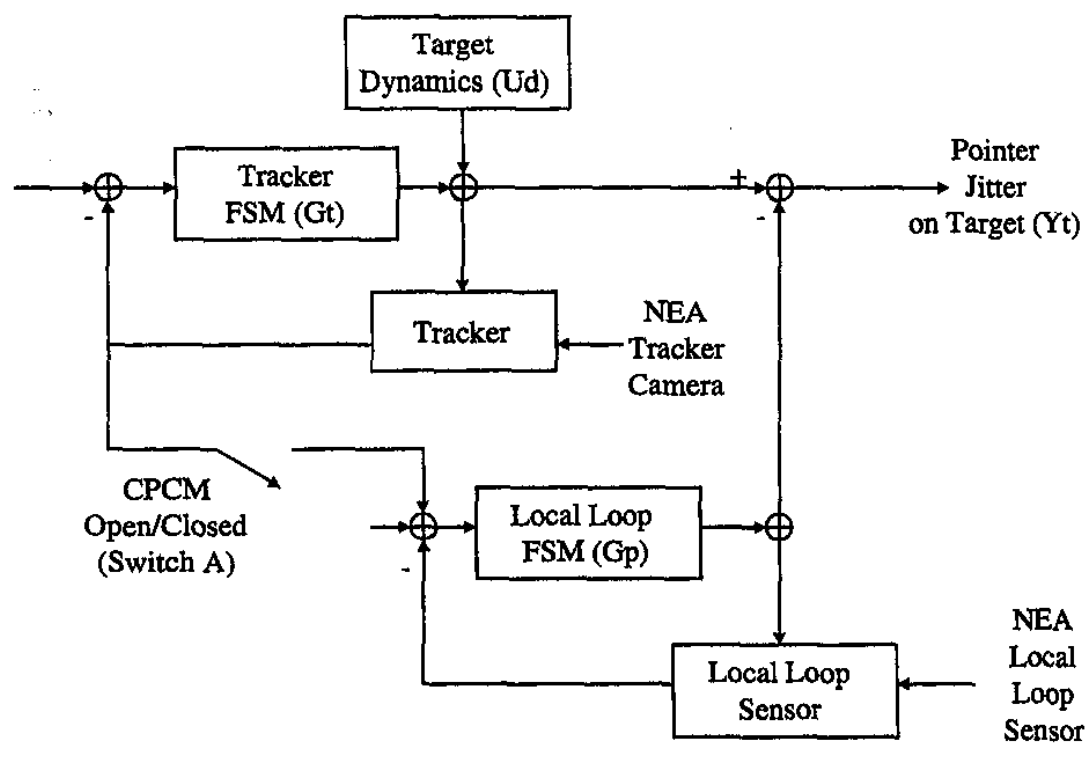

Figure 3. CPCM Block Diagram 
Performance of CPCM tracking is superior to conventional approach.

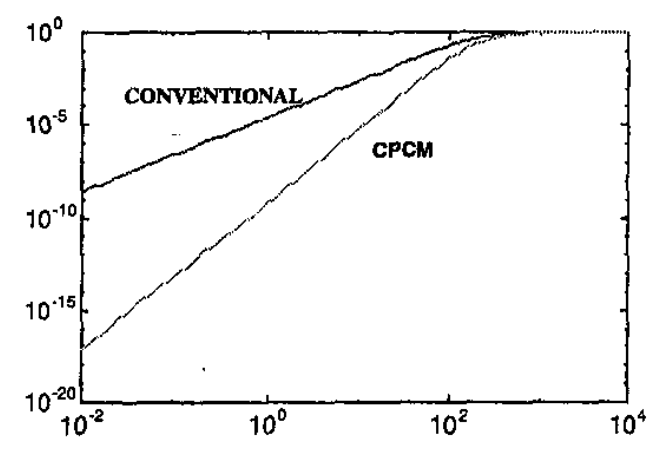

Error Rejection Functions for Type I Tracking and CPCM, $200 \mathrm{~Hz}$ Loops.

CPCM gives Type II error rejection with no overshoot.

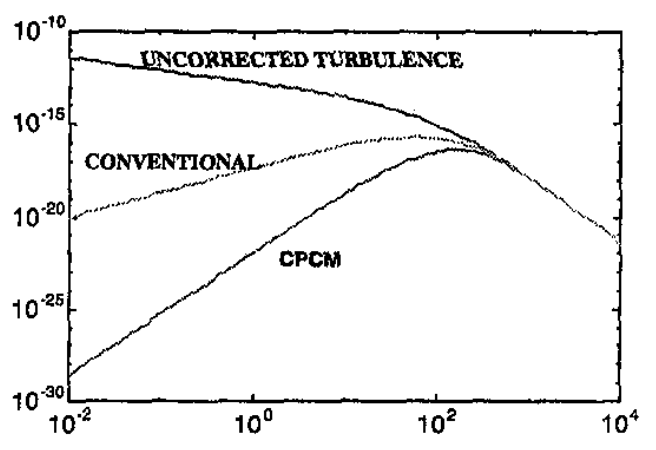

PSDs for Turbulence Tilt and Residual after Type I and CPCM Correction, NOP to Aircraft Target, $200 \mathrm{~Hz}$ Loops.

$$
\begin{aligned}
& \text { RMS tilt: Uncorrected }=1.81 \mu \mathrm{rad} \\
& \text { Type I Compensation }=0.29 \mu \mathrm{rad} \\
& \text { CPCM Compensation }=0.17 \mu \mathrm{rad} .
\end{aligned}
$$

Figure 4. CPCM Simulation Results

closed-loop control. Notice that the non-CPCM system appears to contain only one free integrator in the loop. The CPCM system appears to have type II error rejection - i.e., the closed-loop response is second order. Notice that this was obtained without any double integrators in any of the individual control loops. Simply put, it is a combination of two control loops working in a pseudo "inner loop, outer loop" phenomena.

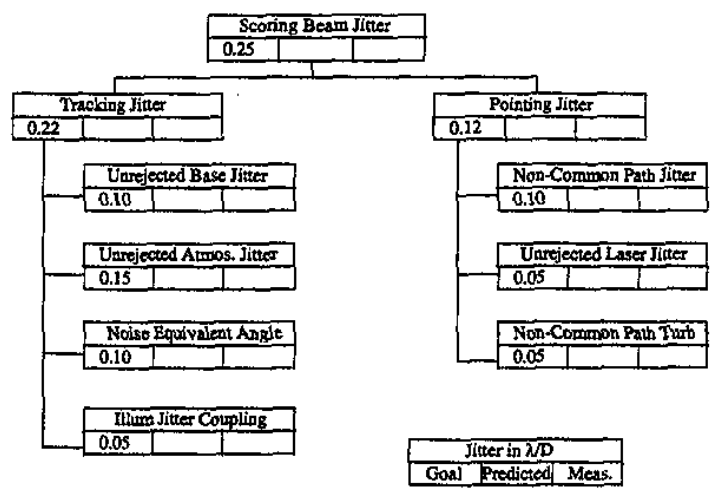

Figure 5. Representative Tracking Budget Including CPCM

\section{REPRESENTATIVE REQUIREMENTS}

In FY1996, the US Air Force Research Laboratory (AFRL) initiated the Airborne Laser (ABL) Advanced Concepts Testbed (ACT) program. A major goal of the program was to successfully demonstrate Acquisition, Tracking and Pointing (ATP) of a Surrogate High Energy Laser (SHEL)/ScrBm down to a quarter of a wavelength RMS tilt error for atmospheric Rytov numbers up to 0.5 . A complete discussion of Rytov numbers can be found in [3]. From this goal, an error budget was created that took into account target track errors and CPCM track errors, with break downs of other errors in each category. Figure 5 shows the error budget for the SHEL ATP.

\section{PROPOSED EXPERIMENT}

To demonstrate the CPCM concept, the AFRL initiated two experiments. The first experiment, a simplified bench top experiment, will demonstrate the type II control performance of CPCM at beam pointing, while the second CPCM experiment will involve the integration of CPCM into the ABL ACT to verify the proposed jitter budget. To date, only the bench top experiment has been fully developed.

The goal of the proposed bench-top experiment will demonstrate the performance associated with $\mathrm{CPCM}$ without concentrating too much on the specific system requirements. The experiment will also be used to check the control software/hardware system common to both CPCM experiments.

Figure 6 shows the proposed bench-top experiment schematic. A $20 \mathrm{~mW}$ helium neon (HeNe) laser will simulate the target return image, while a $5 \mathrm{~mW}$ pumped laser diode greeny $(\lambda=632 \mathrm{~nm})$ will simulate the SHEL. A 0.9 Neutral Density (ND) filter will be placed in front of the HeNe to match the power levels of the two sources. The rest of the setup is similar to that shown in Figure 1. The simulated target beam is reflected off of a FSM, passes through an ASE, and is focused onto a Position Sensing Device (PSD). The simulated SHEL beam first passes 
through the ASE, is split at the ASE, where some of the energy (50\% for this experiment) is reflected off the retroreflector, goes back into the ASE, where the energy is again split by $50 \%$, and is finally focused onto another PSD. Notice that each PSD is proceeded by a spectral filter to filter out any unwanted light from the other source. Also notice that each source also has a $10 \mathrm{X}$ beam expander in front to decrease the spot size at the PSD. The PSDs are simple quadcell detectors with a frequency response greater than $1 \mathrm{KHz}$.

\section{CPCM Hardware Architecture}

The CPCM hardware architecture was born out of ABLACT system requirements that are to be integrated into a complex atmospheric propagation experiment that consisted of multiple subsystems. Figure 7 shows the computer control execution
2. High speed algorithm execution
3. Sensor input
4. Graphical User Interface (GUI)
5. Mirror control output
6. Data logging.
7. Sub-system communication
8. Accurate time

\section{Real-time program execution}

The real-time program execution is performed by a PowerPC, SBC (Single Board Computer) running the realtime, multitasking operating system VxWorks. This combination provides the necessary deterministic response of the system to external and internal events. The heartbeat of the system is controlled by an IRIG-B time card that

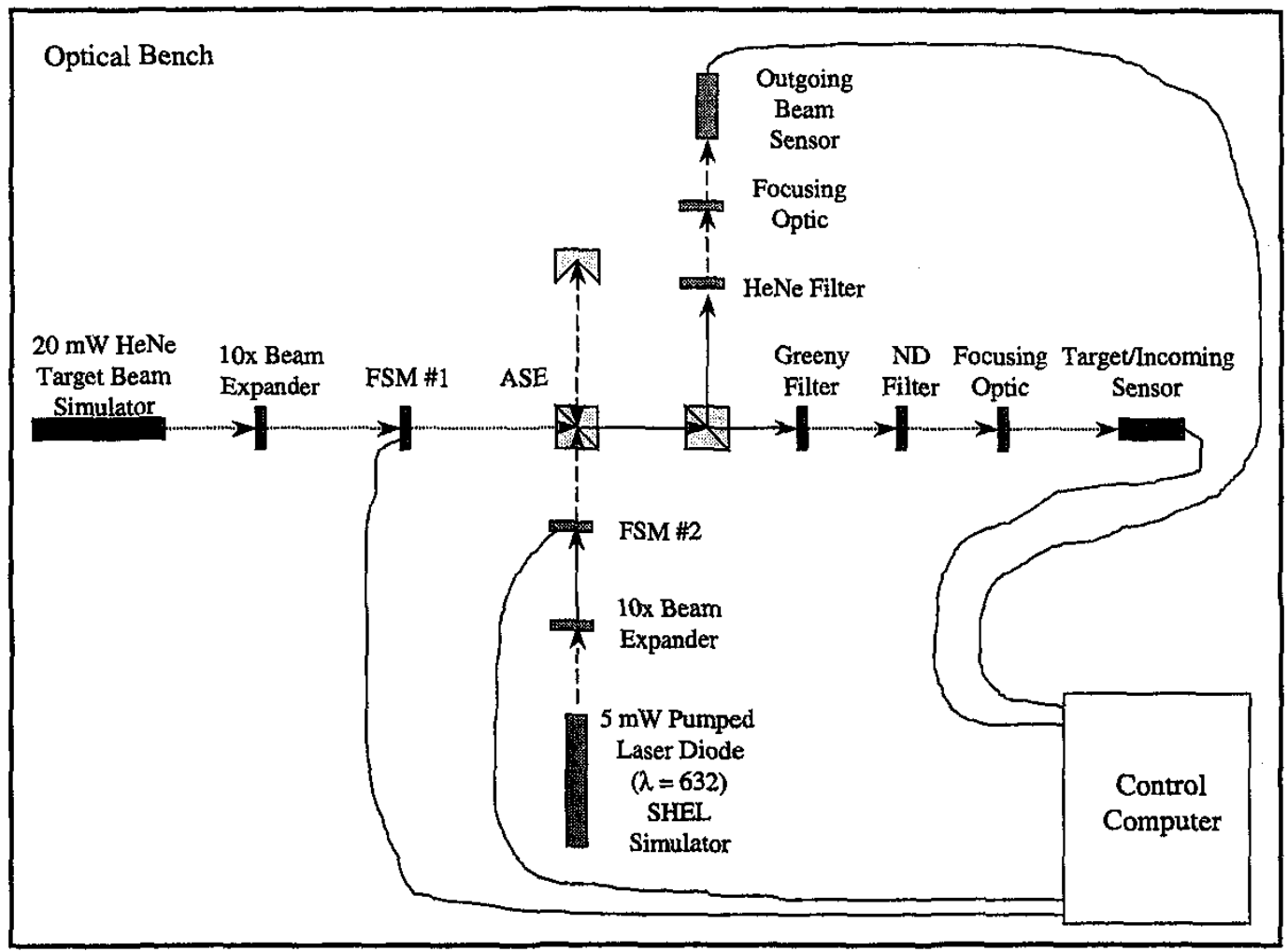

Figure 6. Bench Top Experiment Schematic

architecture for the bench top experiment. These subsystems are a Telescope Gimbal Controller, a Fine and Coarse Video Tracker, an Adaptive Optics System, a Laser Safety System, a Precision Timing System and several Data Reduction Systems.

The CPCM System is a VME-based, COTS implementation that resides in a 21 slot VME chassis. The system can be broken down into the following functions:

1. Real-time deterministic program operation and receives accurate time from a GPS receiver. This card interrupts the VxWorks processor at a precise $1000 \mathrm{~Hz}$ period. VxWorks and the PPC processor provide a very low latency interrupt response time. A $1000 \mathrm{~Hz}$ program task controls the sequence of the remaining operations of the system.

\section{High Speed Algorithm Execution}


The CPCM system receives and processes measured $\mathrm{X}$ and $Y$ directional tilts from several sources. For the full system, these will include the outgoing HEL (High Energy Laser), Scoring Beam, the WFS (Wavefront Sensor) Beacon, the TILL (Tracking Illuminator), the Coarse Plume Tracker, and the Fine Tracker. Each source requires a compensation algorithm to be executed. This task is preformed using a dual i860 processor board from Mercury Computer Systems. The HEL, TILL, and WFS Beacon tilts are provided by an analog to digital converter. The Coarse and Fine Tracker tilts are read into the system from their respective sources via a fiber-optic reflective memory network. filters feed the input of a 16 bit analog to digital converter that is also synchronized by the precise timing strobe from the precision timing system.

\section{Graphical User Interface}

User control of the CPCM system is provided by a graphical user interface running on a WindowsNT workstation. The GUI is written in MATLAB, an analytical data reduction software package from The Mathworks. Communications to the CPCM real-time processor is provided by a $100 \mathrm{Mbit}$, Ethernet link using RPC (Remote Procedure Call) functions.

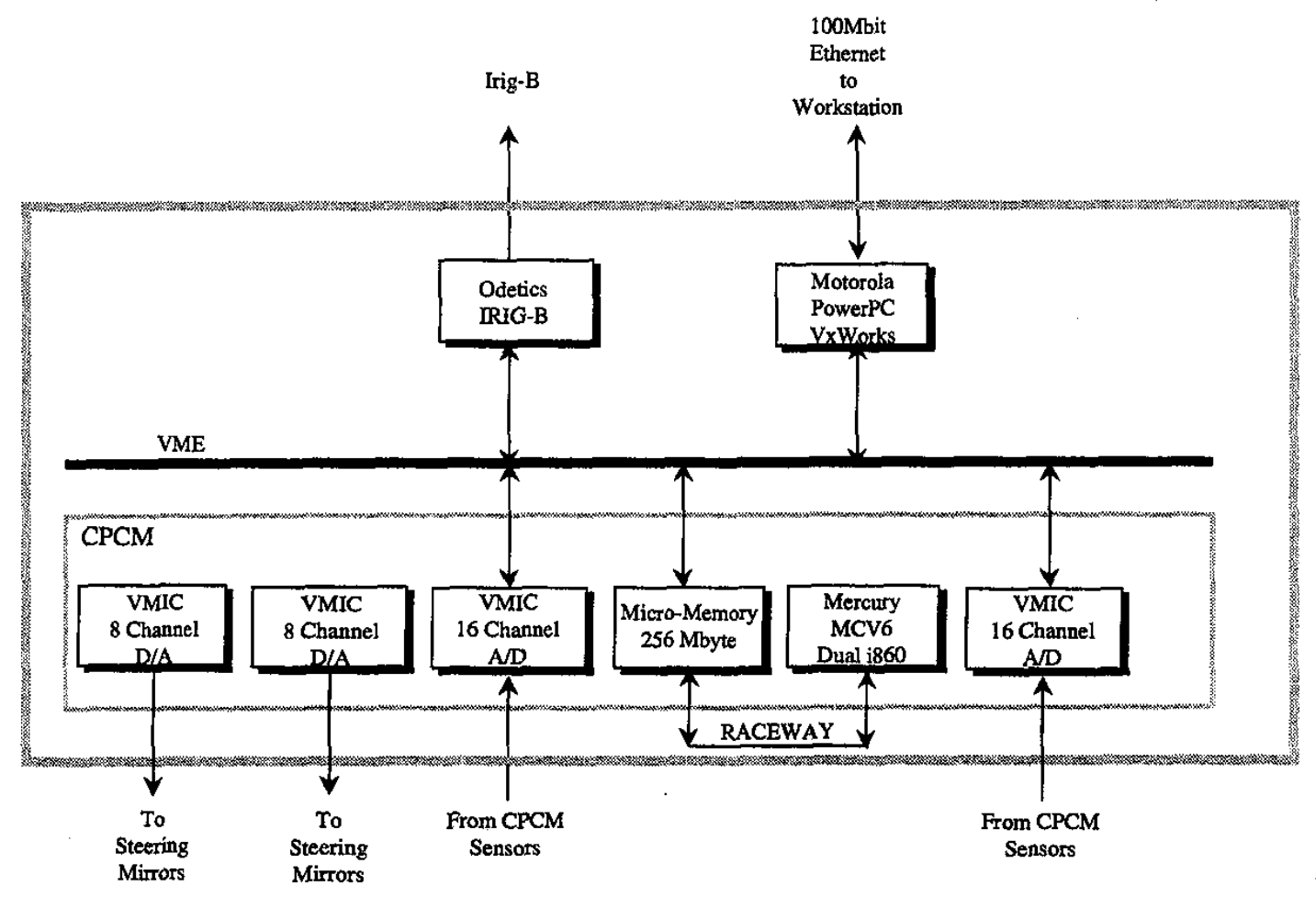

Figure 7. CPCM Computer Control Architecture

\section{Sensor Input}

CPCM requires the measurement of three outgoing tilt sources: one, WFS Beacon, two, the TILL (Track Illuminator), and three, the HEL (High Energy Laser). The HEL is a pulsed laser and as such a system was designed to integrate and capture the energy of the short duration pulses. The precision timing system provides a timing strobe that accurately depicts the time of flight of the beam path. This strobe controls the gating of a combination Integrator/Low Pass Filter. The electronics are allowed to integrate during the duration of the return path of the beam. The filter is then switched to the low pass mode to dump the charge and be ready for the next puise. Each analog output of the sensors is routed through one of these integrator/low pass filters. The

\section{Mirror Control Output}

The CPCM controls several FSMs. The mirror control is provided by a 16 bit, Digital to Analog Converter (DAC). The i860 processors output the algorithm results to the D/A's over the VME bus.

\section{Data Logging}

The CPCM system was designed to save all data necessary to reconstruct events during a time duration of a particular experiment scenario. A 256 Mbyte memory board provides up to 30 minutes of record time. All data logging is controlled by the i860's writing data over the high bandwidth data path Raceway to the memory card. The data can then be archived to disk. 


\section{Sub-System Communication}

The CPCM system incorporates a fiber-optic reflective memory board from VMIC to communicate to the other subsystems in the experiment. This memory board allows other systems to write data into their local memory and have it appear in the CPCM memory after only a short latency period. This method provides an efficient means of distributing control and data to all of the experimental subsystems.

\section{Accurate Time}

Accurate time is provided by an IRIG-B compatible time card from Truetime. The IRIG-B input is provided by a GPS receiver. Time can be read to an accuracy of 1 microsecond.

\section{SUMMARY}

A control architecture has been developed that shows Type II performance for laser pointing systems which are referenced to a target error. The basic concepts have been developed along with the theoretical basis for how the system works. Simulation results have also been shown that verify performance improvements over conventional laser pointing systems. The hardware and software setup for a bench-top experiment has also been presented that will verify the performance shown in simulation and theory.

\section{REFERENCES}

[1] Voelz, David, et al. "Floodbeam Experiment I: Final Report", USAF Phillips Lab Technical Report Number PL-TR-96-1162, Kirtland AFB, NM, 1996

[2] "Aircraft Based Laser (ABL) Risk Reduction", USAF Phillips Lab, Lockheed Martin Missile and Space Technical Interchange Meeting, November 1992.

[3] Goodman, "Statistical Optics", McGraw Hill, New York, 1990. 